\title{
Device management of arrhythmias after Fontan conversion
}

\author{
Sabrina Tsao, MD, ${ }^{\text {a,c }}$ Barbara J. Deal, MD, ${ }^{\text {a,c }}$ Carl L. Backer, MD, ${ }^{\text {b,d }}$ Kendra Ward, MD,,${ }^{\text {a,c }}$ \\ Wayne H. Franklin, MD, ${ }^{\mathrm{a}, \mathrm{c}}$ and Constantine Mavroudis, $\mathrm{MD}^{\mathrm{e}}$
}

Objectives: We assessed our pacemaker strategy, use of antitachycardia therapies, generator longevity, and need for programming changes in patients having Fontan conversion with arrhythmia surgery.

\begin{abstract}
Methods: Between 1994 and 2008, of 121 consecutive patients having Fontan conversion and arrhythmia surgeries, 120 patients underwent pacemaker implantation at the time of Fontan conversion (mean age $22.9 \pm 8.1$ years). Prior pacemakers were in place in 32/120 (26.7\%) patients. Between 1994 and 1998, single-chamber atrial antitachycardia pacemakers were implanted $(n=12)$; atrial rate-responsive pacemakers $(n=31)$ were implanted between 1998 and 2002. Dual-chamber rate-responsive pacemakers $(n=16)$ were used between 2002 and 2003, and subsequently dual-chamber antitachycardia pacemakers $(\mathrm{n}=61)$ have become the pacemaker of choice. Leads have evolved from transatrial endocardial leads to epicardial unipolar and subsequently bipolar leads.
\end{abstract}

Results: Among 87 patients with adequate follow-up, all are currently atrially paced at a minimum lower rate $\geq 70$ beats per minute. Single-chamber atrial pacemakers were implanted in 43 (antitachycardia in 12), and dual-chamber pacemakers in 77 (antitachycardia in 61); multisite ventricular leads were placed in 7 patients. Far-field R-wave sensing in $78.6 \%$ of unipolar atrial leads led to use of epicardial bipolar leads. Late atrioventricular block (24\%) led to routine implantation of dual-chamber pacemakers. Antitachycardia pacing was utilized in $7 \%$. One patient required acute lead revision and 4 required late upgrade to dual-chamber pacemakers. There was no pacemakerrelated infection. Twenty patients required generator change, and the mean device longevity was 7.53 years.

Conclusions: Customized pacemaker therapy can optimize management of patients following Fontan conversion. Device longevity is excellent. Based on our experience with 120 Fontan conversions, we recommend placement of a dual-chamber antitachycardia pacemaker with bipolar steroid-eluting epicardial leads in all patients at the time of the conversion.

The development of chronotropic incompetence following Fontan-type repairs has been shown to correlate with the development of atrial tachycardia. ${ }^{1-3}$ Fontan conversion with limited right atrial ablation has been shown to decrease the incidence of tachycardia, but approximately $25 \%$ in an initial series experienced recurrent atrial tachycardia. ${ }^{4}$ The more extensively modified right atrial maze significantly reduced the incidence of recurrent tachycardia., ${ }^{5,6}$ These strategies incorporated antibradycardia pacing at physiologic rates in an attempt to limit the occurrence of atrial ectopy and subsequent degeneration into atrial tachycardia, and a small percentage of patients used antitachycardia pacing protocols to treat tachycardia recurrences. Epicardial pacemaker implantation can be performed safely in patients having Fontan repairs, but the technological challenges and

\footnotetext{
From the Divisions of Cardiology a and Cardiovascular-Thoracic Surgery, ${ }^{\mathrm{b}}$ Children's Memorial Hospital, and the Departments of Pediatrics ${ }^{\mathrm{c}}$ and Surgery, ${ }^{\mathrm{d}}$ Northwestern University Feinberg School of Medicine, Chicago, IIl; and Center for Pediatric and Congenital Heart Diseases, Cleveland Clinic Children's Hospital, Cleveland Clinic Lerner School of Medicine, ${ }^{\mathrm{e}}$ Case Western Reserve University, Cleveland, Ohio.

Received for publication June 13, 2008; revisions received Oct 13, 2008; accepted for publication Nov 24, 2008; available ahead of print June 18, 2009

Address for reprints: Sabrina Tsao, MD, Division of Cardiology-M/C \#21, Children's Memorial Hospital, 2300 Children's Plaza, Chicago, IL 60614 (E-mail: stsao@ childrensmemorial.org).

J Thorac Cardiovasc Surg 2009;138:937-40

$0022-5223 / \$ 36.00$

Copyright (c) 2009 by The American Association for Thoracic Surgery

doi:10.1016/j.jtcvs.2008.11.066
}

epicardial pacing system longevity in older patients with single ventricle physiology has not been previously reported. ${ }^{7}$ We reviewed the usage and longevity of pacing systems in 120 consecutive patients having Fontan conversion with arrhythmia surgery, including changing device selection, the development of atrioventricular (AV) block, and the utilization and efficacy of antitachycardia modalities.

\section{METHODS}

This was a retrospective, single-center, uncontrolled study and was reviewed and approved by the Institutional Review Board at Children's Memorial Hospital. The charts of 120 consecutive patients having Fontan conversion with arrhythmia surgery and pacemaker implantation at Children's Memorial Hospital between December 1994 and April 2008 were reviewed; 1 additional patient during this time period did not have pacemaker implantation. All patients had functionally single-ventricle physiology: 98 had a dominant left ventricle, 15 had a dominant right ventricle, and 7 had complex single-ventricle anatomy. The median age at the time of their first Fontan operation was 5.8 years (range, 1.1-35 years), at the time of Fontan conversion was 21.6 years (range, 2.6-47.3 years); the median postoperative interval was 15.8 years after the first Fontan operation. Patients had a mean of 1.5 and median of 1 prior midline sternotomies. Prior pacing systems had been implanted in 32/120 (26.7\%) patients, for indications of sinus bradycardia and antiarrhythmic drug usage in 24 , antitachycardia pacing in 6 , and $\mathrm{AV}$ block in 2 patients.

\section{Lead Selection and Implantation Technique}

The type of pacemaker lead that we used evolved over time as technological advances provided new alternatives. Initially, bipolar endocardial leads 


\author{
Abbreviations and Acronyms \\ AAIR = atrial rate-responsive pacemakers \\ AAIT $=$ atrial antitachycardia pacemakers \\ $\mathrm{AV}=$ atrioventricular \\ DDDR $=$ dual-chamber rate-responsive \\ pacemakers \\ DDDRT $=$ dual-chamber anti-tachycardia \\ rate responsive pacemakers
}

were used as epicardial leads but had poor lead longevity. When epicardial steroid-eluting leads were produced, the longevity of epicardial leads became acceptable. Most recently, when bipolar epicardial leads were manufactured, with superior sensing and pacing capabilities, these became the leads of choice. Between 1994 and 1998, atrial lead implantation was performed using transatrially placed endocardial steroid-eluting bipolar tine or screw-in leads (Medtronic 4524, 4024, and 4068; Minneapolis, Minn) in 15 patients. ${ }^{8}$ The transatrial endocardial screw-in lead was placed through a purse-string suture through the atrial wall into either the right or left atrial appendage. Between 1998 and 2003, atrial leads were implanted epicardially using unipolar steroid-eluting leads (Medtronic 4965) in 21 patients; after 2003, bipolar steroid-eluting leads were used (Medtronic 4968) in 84 patients. A total of 21 unipolar and 99 bipolar atrial leads were implanted. With epicardial placement, the atrial lead was placed on the native right atrium anterior to the atriotomy. The left atrial dome was used when atrial thresholds less than $1.5 \mathrm{~V}$ were not obtainable on the right atrium. Epicardial steroid-eluting bipolar ventricular leads were placed on the diaphragmatic aspect of the single ventricle. With ventricular thresholds greater than 1.5 $\mathrm{V}$, a "scored" technique was used: adipose tissue was dissected and removed, allowing placement of the standard bipolar epicardial leads directly on the myocardium. When the scored technique did not achieve thresholds less than $1.5 \mathrm{~V}$ with the epicardial bipolar lead, 2 screw-in non-steroid-eluting unipolar leads (Medtronic 5071) were implanted using a stab technique into the myocardium. The 2 screw-in unipolar leads were then connected with a Y-adapter for bipolar configuration. Whenever possible, multisite ventricular pacing leads (bipolar steroid-eluting) were placed in patients with either complete AV block or patients whose ventricular function was significantly depressed preoperatively or during warming. The additional ventricular lead for multisite pacing was placed on the anterior superior aspect of the single ventricle to allow maximal distance between the 2 ventricular leads.

The pacing strategy included atrial pacing above the intrinsic junctional rate, at 110 to 120 beats per minute, in the immediate postoperative period, and this was incrementally decreased in the first few days following surgery. Chronic pacing was routinely set at a minimum of 70 to 80 beats per minute.

Chronic anticoagulation with warfarin was used in all patients for at least 1 year postoperatively because of the newly placed polytetrafluoroethylene tube graft (W.L. \& Associates Inc, Flagstaff, Ariz) from the inferior vena cava to the pulmonary artery for the extracardiac Fontan. Patients with transatrial endocardial pacing leads remained anticoagulated indefinitely, maintaining international normalized ratio between 2 and 2.5 .

\section{Pacemaker Selection}

The type of pacemaker selected evolved over time, depending on pacemaker availability and expected arrhythmia recurrence. Initially singlechamber atrial antitachycardia pacemakers (AAIT; Intermedics Intertach; Sulzer Intermedics, Winterthur, Switzerland) were used. This pacemaker was not available after 1998, and atrial rate-responsive pacemakers (AAIR) were implanted between 1998 and 2002 (Medtronic Thera and Kappa models). Beginning in 2002, dual-chamber rate-responsive pacemakers (DDDR) were implanted to minimize the impact of far-field R- wave sensing, and the potential for late AV node dysfunction. Since 2003, dual-chamber antitachycardia rate responsive pacemakers (DDDRT) were implanted as these devices became available (Medtronic AT 500, EnRhythm). All dual-chamber pacemakers were programmed in atrial rate-responsive pacing mode, with the exception of patients with complete or advanced AV block. An epicardial defibrillator (Medtronic Gem III AT) was implanted using a medium epicardial defibrillator patch (Medtronic 6721 ) on the anterior ventricular surface in 1 patient for secondary prevention following resuscitation from ventricular tachycardia.

\section{Lead and Device Assessment}

Lead characteristics were assessed at the time of implantation. Pacing thresholds were tested at 0.5 -millisecond pulse width and were assessed as low $(<1.5 \mathrm{~V})$, medium $(1.5-2.5 \mathrm{~V})$, and high $(>2.5 \mathrm{~V})$. Far-field Rwave sensing was defined as significant if the $\mathrm{R}$ wave detected by the atrial lead was greater than $0.75 \mathrm{mV}$. Pacing complications were classified as lead dislodgement or acute postoperative pacing thresholds greater than $3.5 \mathrm{~V}$.

Follow-up data were assessed from patient records or through follow-up letters from referring physicians. Lead characteristics during follow-up were assessed using threshold testing and/or programmed parameters; programming at $<3.5 \mathrm{~V}$ at 0.4 milliseconds was considered as a low threshold. Device longevity was assessed as the time to generator replacement. The need for dual-chamber pacing and antitachycardia pacing utilization and efficacy was recorded.

Statistical analysis was performed using Fischer exact test.

\section{RESULTS}

Acute implantation data were assessed in all 120 patients. However, follow-up data were available in a total of $87 \mathrm{pa}-$ tients. Thirty-three patients are excluded from data analysis (in 19 patients, follow-up pacing data were not available as they were referred from overseas and other institutions; 6 patients had orthotopic heart transplantation, and 8 patients died during follow-up). There was only 1 perioperative mortality unrelated to pacing issues.

Thirty-two patients had prior implantation of pacing systems: transvenous atrial pacemaker in 9 (3 antitachycardia, 6 rate-responsive), and 23 patients with epicardial pacemakers (atrial in 4, ventricular in 5, and dual-chamber in 14 patients). At the time of surgery, significant atrial thrombi were identified on 3/9 (33.3\%) transvenous leads; 1 patient had a major cerebrovascular accident with resultant hemiplegia.

\section{Acute Lead Implantation Thresholds}

Pacing thresholds for atrial leads were low in $98(81.7 \%)$ and medium in $22(18.3 \%)$ patients. In dual-chamber devices, ventricular thresholds were low in $42(54.5 \%)$ and medium in $35(45.5 \%)$ patients. It is often very difficult to obtain adequate thresholds in these patients with dense scarring secondary to multiple prior procedures. Acute lead revision was performed in 1 patient $(0.8 \%)$ with a transatrially placed endocardial atrial lead on postoperative day 2 due to intermittent atrial noncapture. There was no significant difference in atrial pacing thresholds between transatrially placed endocardial versus epicardial leads $(P=.07)$ or between unipolar versus bipolar leads $(P=.118$; see Table 1). Transatrial endocardial leads trended to have lower but 
TABLE 1. Lead implantation data

\begin{tabular}{lccccc}
\hline & $\begin{array}{c}\text { No. of } \\
\text { leads }\end{array}$ & $\begin{array}{c}\text { Low } \\
\text { threshold } \\
(<\mathbf{1 . 5} \mathbf{~ V )}\end{array}$ & $\begin{array}{c}\text { Medium } \\
\text { threshold } \\
(\mathbf{1 . 5 - 2 . 5} \mathbf{~ V )}\end{array}$ & $\begin{array}{c}\text { High } \\
\text { threshold } \\
(>\mathbf{2 . 5} \mathbf{~ V )}\end{array}$ & $\boldsymbol{P}$ value \\
\hline Atrium & & & & & \\
$\quad$ Transatrial & 15 & $15(100 \%)$ & 0 & 0 & \\
Epicardial & 105 & $83(79 \%)$ & $17(16.2 \%)$ & $5(4.8 \%)$ & .07 \\
Unipolar & 21 & $20(95.2 \%)$ & $1(4.8 \%)$ & 0 & \\
Bipolar & 99 & $78(78.8 \%)$ & $16(16.2 \%)$ & $5(5.1 \%)$ & .118 \\
Ventricle & & & & & \\
$\quad \begin{array}{l}\text { Unipolar } \\
\text { Bipolar }\end{array}$ & 21 & $10(47.6 \%)$ & $7(33.3 \%)$ & $4(19 \%)$ & \\
\hline
\end{tabular}

not statistically significant acute implantation thresholds (see Table 3). For ventricular leads, there was no statistical difference in pacing thresholds between screw-in non-steroid-eluting unipolar versus epicardial steroid-eluting bipolar leads $(P=.455$; see Table 1$)$.

\section{Devices and Pacing Modes}

Pacing modes are summarized in Table 2. Among the 77 patients with dual-chamber pacemakers (DDDR and DDDRT), 19 (24.7\%) patients utilized dual-chamber pacing mode acutely postoperatively. One patient had preexisting complete AV block, 9 had acquired complete AV block (3 transient), and $9(7.5 \%)$ had marked AV node dysfunction during amiodarone infusion.

\section{Multisite Pacing}

Multisite ventricular pacing leads were placed in 7 patients, with utilization of multisite pacing in 4 patients. One patient had preexisting complete AV block, 2 had acquired complete $\mathrm{AV}$ block, and 1 patient had significantly depressed ventricular function preoperatively. Multisite pacing effectively reduced QRS duration only in the 1 patient with reduced ventricular function; ventricular function improved postoperatively, but the relationship to multisite pacing was not clear.

\section{Follow-up}

Adequate pacemaker data were available for 87 patients. For patients with atrial pacing systems, the median followup period was 83 months, and for patients with dual-chamber pacemakers, the median follow-up was 32 months. Six patients $(7.5 \%)$ take chronic antiarrhythmic medications at mean follow-up of 46.3 months.

\section{Pacing Therapies}

Atrial antitachycardia pacing was utilized effectively for termination of recurrent atrial tachycardia in 5/73 $(6.8 \%)$ patients ( 3 with AAIT pacemakers and 2 with DDDRT pacemakers). Rate-responsive pacing was utilized in all patients with such devices; rarely, patients requested this programming off due to the sensation of palpitations. All patients use atrial antibradycardia pacing to maintain atrial
TABLE 2. Pacing mode at follow-up pacemaker interrogation

\begin{tabular}{|c|c|c|c|c|}
\hline \multirow[b]{2}{*}{ Device } & \multirow[b]{2}{*}{$\mathbf{n}$} & \multicolumn{2}{|c|}{ Pacing mode } & \multirow{2}{*}{$\begin{array}{c}\text { Median } \\
\text { follow-up (mo) }\end{array}$} \\
\hline & & AA A I & DDD & \\
\hline \multicolumn{5}{|l|}{ Single-chamber } \\
\hline $\begin{array}{l}\text { Atrial } \\
\text { antitachycardia }\end{array}$ & 12 & 12 & & 144 \\
\hline $\begin{array}{l}\text { Atrial rate- } \\
\text { responsive }\end{array}$ & 31 & 31 & $\begin{array}{l}4 \text { (later upgrade } \\
\text { to DDD) }\end{array}$ & 93 \\
\hline \multicolumn{5}{|l|}{ Dual-chamber } \\
\hline Rate-responsive & 16 & 13 & 3 & 72 \\
\hline Antitachycardia & 61 & 49 & 13 & 25 \\
\hline $\begin{array}{l}\text { Multisite } \\
\text { antitachycardia }\end{array}$ & $7 *$ & 3 & $4 *$ & 39 \\
\hline Multisite + defibrillator & 1 & 0 & 1 & $46(\exp )$ \\
\hline
\end{tabular}

rates greater than 70 beats per minute. Among 77 patients receiving dual-chamber pacemakers, 16/77 (20.8\%) patients required dual-chamber pacing during chronic follow-up due to the development of advanced AV block. Among the 43 patients with single-chamber pacemakers (AAIT and AAIR), $4(9.3 \%)$ patients required late upgrade to DDD pacemaker: 2 patients developed late AV block and 2 patients to differentiate far-field $\mathrm{R}$-wave sensing versus recurrent atrial tachycardia. Overall, dual-chamber pacing was utilized in 20/84 (24\%) patients with adequate follow-up data.

\section{Unipolar Versus Bipolar Atrial Leads}

Far-field R-wave sensing was present in 11/14 (78.6\%) patients with unipolar atrial leads and 16/73 (21.9\%) patients with bipolar atrial leads $(P<.0001)$ (Table 3$)$.

\section{Pacemaker Longevity}

Pacemaker replacement has been performed in 20/87 $(23 \%)$ patients. Among these patients, the mean generator longevity was 7.53 years. No patient required late lead revision due to fracture. One patient with heterotaxy syndrome, preoperative AV block, permanent atrial fibrillation, and advanced ascites required revision of the ventricular lead 10 months postoperatively due to high pacing thresholds.

\section{DISCUSSION}

Optimizing pacing strategies was a challenging but important adjunctive therapy in this series of 120 patients having Fontan conversion with arrhythmia surgery. Despite multiple

TABLE 3. Follow-up FFR sensing in atrial leads

\begin{tabular}{lcc}
\hline $\begin{array}{c}\text { Atrial leads/ } \\
\text { thresholds }\end{array}$ & $\begin{array}{c}\text { Negligible FFR } \\
(<\mathbf{0 . 7 5} \mathbf{~ m V})\end{array}$ & $\begin{array}{c}\text { Significant FFR } \\
(>\mathbf{0 . 7 5} \mathbf{~ m V})\end{array}$ \\
\hline Unipolar & 3 & 11 \\
Bipolar & $57^{*}$ & 16 \\
\hline
\end{tabular}

$F F R$, Far-field $\mathrm{R}$ wave. $* P<.0001$. 
prior surgeries, debilitating arrhythmias, and chronic antiarrhythmic therapy, epicardial leads were implanted with low or medium threshold characteristics in all patients. Antibradycardia atrial pacing optimizes cardiac output and, we believe, minimizes the opportunities for atrial ectopy precipitating tachycardia. ${ }^{2}$ Significant atrial thrombi were present on $3 / 9$ previously placed transvenous leads. We initially used transatrial placement of endocardial leads; the availability of steroid-eluting leads combined with thrombus seen on existing transvenous leads led to the abandonment of transatrial lead placement. Acute lead revision was necessary in only 1 patient, and no patient required reoperation for lead fractures. Lead placement at the time of surgery is challenging, but these results are encouraging. The changing patterns of device availability, a small but present incidence of recurrent atrial tachycardia, and technical limitations based on anatomy required an evolving approach to device selection.

The utilization of bipolar atrial leads significantly reduced the incidence of far-field R-wave sensing and the resultant problems in tachycardia recognition. Antitachycardia atrial pacing therapies were utilized in $6.8 \%$ of patients, reflecting a decrease in atrial tachycardia with changing arrhythmia surgery, but underscoring the potential for late arrhythmia occurrence. This may be low, but it is very important. The cost of the dual-chamber antitachycardia pacemaker is less than a standard dual-chamber pacemaker.

Based on the need for dual-chamber pacing in up to $21 \%$ of patients during midterm follow-up and the need for a sternotomy or thoracotomy for new lead placement, it has become our standard practice to place epicardial ventricular leads on all patients.

Ventricular lead placement could be challenging, requiring either a "scored" or "stab" technique to achieve adequate pacing thresholds in a small number of patients. This is due to the amount of epicardial adipose tissue present and the scar tissue from prior procedures.

Patients who had limited modified right atrial maze surgery in the beginning of the series had a higher incidence of recurrent atrial tachycardia ${ }^{4}$ and have successfully used atrial antitachycardia pacing to terminate tachycardias. With increasing complexity of anatomy and arrhythmia subsets, antitachycardia pacing may become a more important part of chronic recurrent atrial arrhythmia management. ${ }^{9}$ Patients with preexisting complete heart block or patients at risk of developing complete heart block (L-transposition-type anatomy) may benefit from multisite pacing. There are a small number of patients who developed marked AV block during the initial postoperative intravenous amiodarone infusion and required transient dual-chamber pacing. In our series, the number of patients using multisite pacing was too small and follow-up was too short to comment on eventual ventricular function outcome. Achieving optimal thresholds is important to avoid the risk of repeat sternotomies. Customized pacemaker therapy can optimize management of patients following Fontan conversion. Based on our experience with 120 Fontan conversions, we recommend placement of a dual-chamber antitachycardia pacemaker with bipolar steroid-eluting epicardial leads in all patients at the time of the conversion.

There are several limitations of our study. This was a single-center, retrospective uncontrolled study. All the patients were operated on by the same 2 surgeons. The main limitation of our study is the limited availability of complete follow-up pacemaker parameters and outcomes. Because most patients are referred from out of region and frequently transition from pediatric to adult cardiologists, follow-up data frequently rely on communications from patients.

Resynchronization therapy for single-ventricle patients needs to be assessed with multicenter studies due to the small number of patients at each institution.

\section{References}

1. Dilawar M, Bradley SM, Saul JP, Stroud MR, Balaji S. Sinus node dysfunction after intraatrial lateral tunnel and extracardiac conduit Fontan procedures: a study of 24-hour Holter recordings. Pediatr Cardiol. 2003;24:284-8.

2. Fishberger SB, Wernovsky G, Gentles TL, Gauvreau K, Burnett J, Mayer JE Jr, et al. Factors that influence the development of atrial flutter after the Fontan operation. J Thorac Cardiovasc Surg. 1997;113:80-6.

3. Fontan F, Baudet E. Surgical repair of tricuspid atresia. Thorax. 1971;26:240-8.

4. Deal BJ, Mavroudis C, Backer CL, Buck SH, Johnsrude C. Comparison of anatomic isthmus block with the modified right atrial Maze procedure for late atrial tachycardia in Fontan patients. Circulation. 2002;106:575-9.

5. Deal BJ, Backer CL, Ward KM, Tsao S, Dechert B, de Freitas A, Franklin WH, Mavroudis C. Medical characteristics and midterm outcome: 100 consecutive Fontan conversions with arrhythmia surgery [abstract]. Circulation. 2007;116(suppl II):II-414 (Abstract 1923).

6. Mavroudis C, Backer CL, Deal BJ, Johnsrude C, Strasburger J. Total cavopulmonary conversion and maze procedure for patients with failure of the Fontan operation. J Thorac Cardiovasc Surg. 2001;122:863-71.

7. Cohen MI, Vetter VL, Wernovsky G, Bush DM, Gaynor JW, Iyer VR, et al. Epicardial pacemaker implantation and follow-up in patients with a single ventricle after the Fontan operation. J Thorac Cardiovasc Surg. 2001;121:804-11.

8. Hoyer MH, Beerman LB, Ettedgui JA, Park SC, del Nido PJ, Siewers RD. Transatrial lead placement for endocardial pacing in children. Ann Thorac Surg. 1994;58: 97-102.

9. Deal BJ, Mavroudis C, Backer CL. Beyond Fontan conversion: surgical therapy of arrhythmias including patients with associated complex congenital heart disease. Ann Thorac Surg. 2003;76:542-53. 